\title{
Development and Field Testing of a Spatial Decision Support System to Control Populations of the European Cherry Fruit Fly, Rhagoletis cerasi, in Commercial Orchards
}

\author{
Charalampos S. Ioannou ${ }^{1,+}$, Stella A. Papanastasiou ${ }^{1,+} \mathbb{1}$, Kostas D. Zarpas $^{1} \mathbb{1}$, \\ Miguel Angel Miranda ${ }^{2}$, Andrea Sciarretta ${ }^{3}$, David Nestel ${ }^{4}$ and Nikos T. Papadopoulos ${ }^{1, * \text { (D) }}$ \\ 1 Laboratory of Entomology and Agricultural Zoology, Department of Agriculture Crop Production and Rural \\ Environment, University of Thessaly, Fytokou St., 38446 N. Ionia (Volos) Magnisia, Greece; \\ ioannoubabis@yahoo.com (C.S.I.); stelapap@hotmail.com (S.A.P.); kzarp@uth.gr (K.D.Z.) \\ 2 Applied Zoology and Animal Conservation Research Group, University of the Balearic Islands, \\ UIB-INAGEA, 07122 Palma de Mallorca, Spain; ma.miranda@uib.es \\ 3 Department of Agriculture, Environmental and Food Sciences, University of Molise, via De Sanctis, 86100 \\ Campobasso, Italy; sciarretta@unimol.it \\ 4 Department of Entomology, Institute of Plant Protection, ARO, Rishon Letzion P.O. Box 15159, Israel; \\ nestel@volcani.agri.gov.il \\ * Correspondence: nikopap@uth.gr; Tel.: +30-24210-93285 \\ + The authors contributed equally to this article.
}

Received: 31 August 2019; Accepted: 18 September 2019; Published: 20 September 2019

\begin{abstract}
The European cherry fruit fly, Rhagoletis cerasi (Diptera: Tephritidae), is a key pest for the cherry production industry in Europe and west Asia that has recently invaded North America. Insecticide applications are frequently employed to control this devastating pest, often without considering its population trends. We developed a novel decision support system (DSS), and field tested it in commercial sweet cherry orchards in central Greece. The DSS includes two algorithms that predict the timing of adult activity in the wild and support pest management decisions, based on $R$. cerasi population trends and pesticide properties, respectively. Preparatory monitoring of the testing area during 2014, using adult traps, revealed high population densities of $R$. cerasi in non-managed sweet cherry orchards and low densities in commercial ones. Implementation of the DSS during 2015 resulted in low $R$. cerasi adult population densities and zero fruit infestation rates in commercial cherry orchards. Similar population and infestation rates were recorded in conventionally treated plots that received on average two insecticide applications compared to the one-half that the DSS treated plots received. Simultaneously, high population densities and fruit infestation rates were recorded in non-managed cherry orchards. Apparently, the implementation of the simple DSS we developed reduces the cost of $R$. cerasi management and minimizes the chemical footprint on both the harvested fruit and the environment.
\end{abstract}

Keywords: precision agriculture; integrated pest management; electronic trap; spatial decision support system; pest management algorithm

\section{Introduction}

The European cherry fruit fly, Rhagoletis cerasi (L.) (Diptera: Tephritidae), is a major pest of both sweet and sour cherries in Europe, the near and middle East, as well as East Asia [1]. For many decades, $R$. cerasi has been the only fruit fly infesting cherry fruits in these regions and the main pest for the cherry production systems in Western Europe and Asia. Invasion of the eastern (North 
American) cherry fly, Rhagoletis cingulata (Loew), has broadened the list of cherry fruit fly pests in several European countries [1,2]. Nonetheless, $R$. cingulata is a rather minor pest of sweet cherries in Europe, causing fruit infestation in late ripening cultivars and mainly in sour cherries [1]. The more recent invasion of Drosophila suzukii (Matsumura) (Diptera: Drosophilidae) into Europe has established a new, much more complicated reality for cherry production, since both $R$. cerasi and D. suzukii are now considered key pests of sweet and sour cherry production in Europe [3]. Hence, there is currently enormous pressure on the sustainability of established Integrated Pest Management (IPM) systems in cherry production since several insecticide applications, close to harvest, are required to assure zero, or very low infestation rates, and zero residue on the fresh fruit that the cherry market demands.

The recent invasion and establishment of R. cerasi in Canada (2016) and the United States of America (2017) has caught considerable attention [4]. This is because ecological modelling (CLIMEX) predicts a wide range of cherry producing areas in the USA (e.g., Washington and California) as suitable for the establishment of the European cherry fruit fly [5]. As a result, most of the cherry cultivation in North America is threatened by an additional fruit fly that may increase the risk of trading and the cost of production. The addition of $R$. cerasi into US cherry production will undoubtedly increase the number and volume of insecticides application with major negative effects on cost, environment and human health.

Rhagoletis cerasi is a univoltine fruit fly species that undergoes, at the pupal stage, a long summer-winter dormancy [6]. Diapause termination is habitat specific and takes place from middle to the end of winter (e.g., January, February). It is followed by a quiescence period of variable duration, in the different areas, that allows pupae to complete development and adults to emerge later in spring $[1,6]$. Usually the initiation of adult activity in the wild, evidenced by adult trapping in yellow sticky traps, coincides with the beginning of the ripening period of sweet cherries. Females of $R$. cerasi initiate egg laying on ripening or ripe fruit, starting at the phenological stage of fruit color break which is very close to harvest. A single egg is deposited in each fruit and high levels of infestation (up to $100 \%$ ) are reported when no interventions are practiced. Predicting the timing of adult activity (i.e., adult emergence) and the population density are crucial to design sound management strategies against this pest.

Management of the European cherry fruit fly in commercial orchards relies mainly on spraying the entire orchard. This decision is assisted by information regarding population trends that determines both the timing and need for interventions [7]. Alternative control tactics that have been proposed and pilot tested include mass trapping, bait spray applications, biological control, exclusion netting and sterile insect releases [8-14]. However, for various reasons and mainly because some of the above alternative control tactics are either ineffective or difficult to implement, currently, only mass trapping is systematically exercised in low input, low intensity production systems, backyards and organic farming [7] as a reliable alternative to pesticide application. Mass trapping systems are based on yellow sticky panels with the provisional inclusion of ammonium bicarbonate as a bait [11,12]. Timing of deployment, adopted density and spatial arrangement of the sticky mass trapping devices are important within the treated area. Adults of the European cherry fruit fly are poor flyers and usually do not disperse over long distances [15]. Hence, they tend to remain within a short distance of the host tree, forming clear clusters. Thus, detecting "hot spots" of R. cerasi populations in commercial orchards will improve our ability to reduce the volume of insecticides utilization by directing the application to the clusters of the pest (i.e., "precision targeting").

Spatial and temporal modelling of fruit fly populations is a key tool to understand the ecology of the species and to design control strategies against these pests. Degree day models to determine $R$. cerasi adult emergence patterns have been proposed $[16,17]$ and used to set the initiation of adult monitoring in commercial orchards. In addition, population dynamics models describing the general temporal patterns of $R$. cerasi population trends have been used for several years to direct management efforts in Switzerland [18]. However, the Sopra platform and other similar approaches provide a generic guidance based on phenological models (http://www.sopra.info). Recent efforts focus on 
modelling the on-farm behavior of females [19] and on computer assisted Integrated Pest Management approaches to control R. cerasi [20].

Algorithms (i.e., "step by step procedures for solving or accomplish a problem") provide the foundation for developing Decision Support Systems to assist IPM and other insect management programs [21]. Despite frequent reference in scientific papers and important developments over the last few decades, Spatial Decision Support Systems (SDSS) have just recently started to be developed and tested, especially to manage fruit flies [22-25], including the European cherry fruit fly [20]. Regarding fruit flies, the most advanced SDSS developed and tested until today is MedCila [22], which has been adapted to support management decisions for the Area Wide (AW) control of the Mediterranean fruit fly in citrus crops in Israel. A more advanced version of MedCila is currently being developed as an application and expected to be commercially tested next year in Israel (Gilad Gefen, Citrus Marketing Board, Fruit and Vegetables Grower's Organization of Israel, personal communication). The system is based on acquisition of experts' knowledge, identification of the criteria and incorporation into a Geographic Information System (GIS). Major criteria that are used by MedCila to assist management decisions include adult trapping, host susceptibility, historic patterns of medfly populations and degree day developmental modelling. Sweet cherry cultivation in most European countries is rather fragmented with medium and small size farms scattered throughout a broader cherry producing area. In such a system a farm-specific Integrated Pest Management system should be implemented that considers within-farm pest population trends, topography, host/cultivar composition, ripening periods and other particularities. Indeed, individual-based bottom up methodologies have been recently considered as the foundation to developing on-farm IPM approaches $[19,20]$. Simulation of the Pest on Farm model was found to provide an acceptable representation of $R$. cerasi population trends and infestation levels under an IPM scenario in a pilot site [19]. Nonetheless, the Pest on Farm platform has not been used yet to assist farmers with pest management decisions for $R$. cerasi or other fruit flies.

The present paper (a) describes a newly developed Spatial Decision Support System for the European cherry fruit fly and (b) presents results of its field testing in a commercial sweet cherry production system in central Greece. The developed algorithm includes two parts: The first targets adult emergence in the wild and defines the timing of deploying adult monitoring systems; the second, which is more elaborated, considers phenological patterns of the cherry cultivation, biological information regarding the population trends of the European cherry fruit fly, ecological traits of its populations, chemical and toxicological characteristics of the insecticide to be selected for control and, most importantly, definition of spots (geographic delimited area) where an insecticidal application needs to be applied. The study also provides initial results of field implementation of the developed SDSS in commercial orchards in central Greece. During the first year of the study, background information of the cherry farms was collected. During the second year, the proposed SDSS was implemented in real commercial farm conditions. Finally, the performance of the developed SDSS was contrasted against IPM-adopted approaches by farmers and unmanaged cherry orchards. The IPM measures already adopted by the cherry producing farmers in Greece include basic population monitoring and control using certified insecticides. The timing of sprayings is based on adult flies captured in traps, agronomist consultation and farmer experience.

\section{Materials and Methods}

\subsection{Test Area and Experimental Orchards}

The experimental farms were located in Agia, Larisa (Thessaly, central Greece), an agricultural area producing mostly pome fruits (apples, pears), stone fruits (peaches, apricots, cherries) and olives. Three commercial cherry orchards located in different sites (approximately 2.5 ha each) were selected for the experiments (Figure 1). Each orchard included several cherry cultivars (Table 1). This common practice allows growers to expand the fruit collection period and therefore to achieve better prices on the market. On the other hand, the temporal divergence of cherry ripening among the different cultivars 
makes the implementation of control strategies against $R$. cerasi rather challenging. The orchards' pruning system involved a linear palmetta formation, i.e., flat supported canopy development. The tree density in all orchards was 1660 trees/ha with a spacing of $4 \mathrm{~m}$ between rows and $1.5 \mathrm{~m}$ between trees. The age of the trees was 4.5 years, the average height of the canopy was $3.25 \mathrm{~m}$ (minimum $2 \mathrm{~m}$ and maximum $4.5 \mathrm{~m}$ depending on the cultivar) and the height of the trunk varied from 0.5 to $0.6 \mathrm{~m}$. A drip irrigation system was used, and no intercropping method was applied in the respective orchards. The average yield was $10 \mathrm{~T} /$ ha (minimum 8 and maximum $12 \mathrm{~T} / \mathrm{ha}$ ).

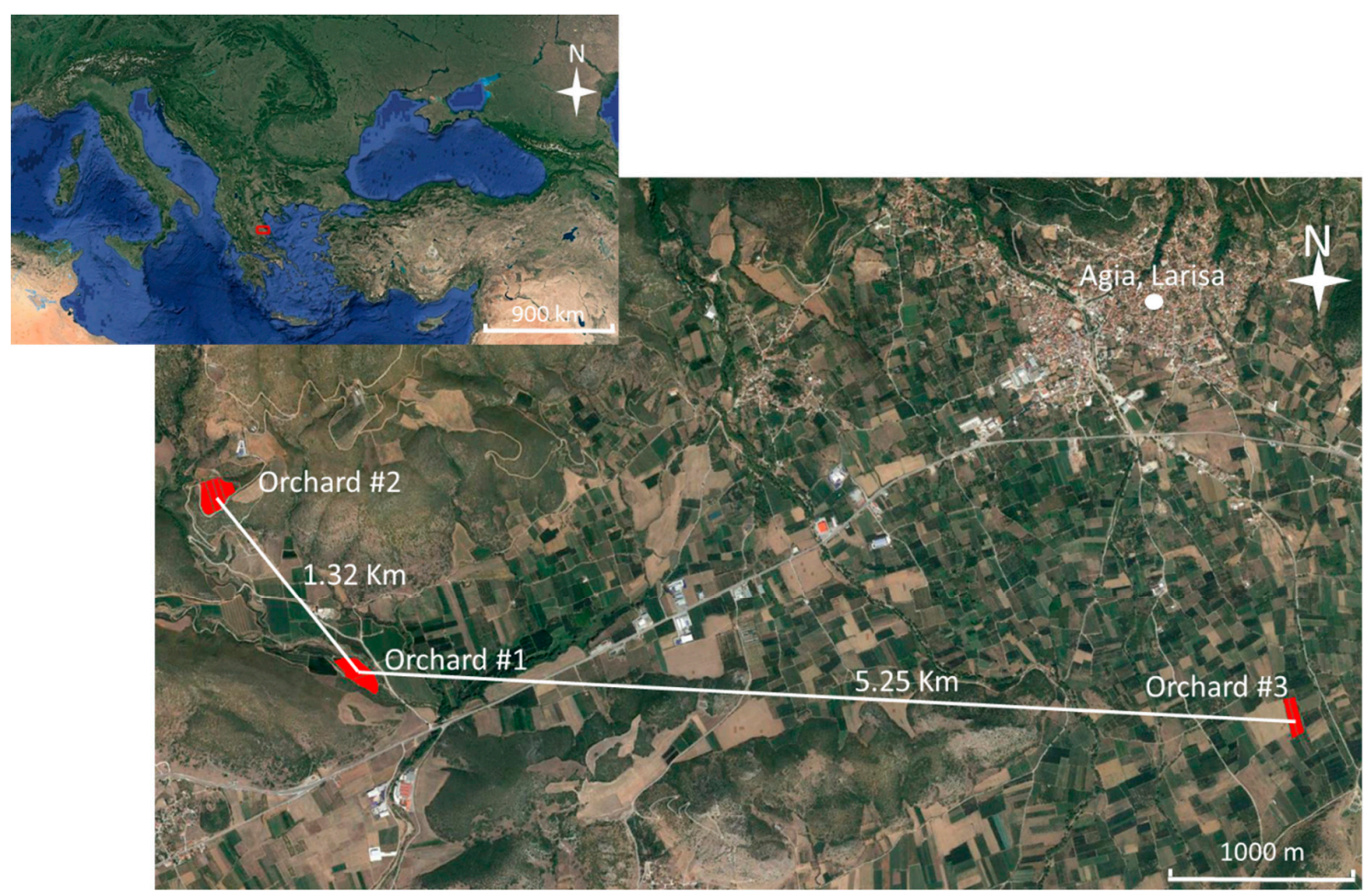

Figure 1. Geographic location of the experimental orchards in relation to the town Agia and distances between them in kilometers (satellite images cropped from Google Earth Pro).

Table 1. Sweet cherry cultivars grown in different experimental plots. "DSS" plots were managed according to the Decision Support System developed, "Conventional" plots were managed according to the IPM-adopted methodology and "Control" plots were managed according to the rules of organic farming. DSS and Conventionally treated plots were situated in Orchards 1, 2 and 3.

\begin{tabular}{|c|c|c|c|c|c|c|c|}
\hline \multirow{2}{*}{$\begin{array}{l}\text { Plots } \\
\text { DSS } 1\end{array}$} & \multicolumn{7}{|c|}{ Sweet cherry (Prunus avium) cultivar } \\
\hline & Ferrovia & Grace star & Lory Bloom & Regina & Skeena & Summit & \\
\hline Conv. 1 & Kordia & Larian & Samba & Sweet early & & & \\
\hline DSS 2 & Regina & Kordia & Ferrovia & & & & \\
\hline Conv. 2 & Giant red & Skeena & & & & & \\
\hline DSS 3 & Giant red & Grace Star & Bakirtzeika & & & & \\
\hline Conv. 3 & Bakirtzeika & Vasiliadi & & & & & \\
\hline DSS 4 & Grace Star & Kordia & Larian & Lory Bloom & Samba & Summit & Sweet early \\
\hline Conv. 4 & Grace Star & Kordia & Larian & Lory Bloom & Samba & Summit & Sweet early \\
\hline Control & Burlat & Bakirtzeika & & & & & \\
\hline
\end{tabular}

Before the initiation of our research activities, no systematic monitoring of R. cerasi was performed to assist the control applications by growers. Instead, IPM-adopted sprayings were exercised every year. Initiation of applications coincided usually with the fruit color break from yellow to reddish, which is the critical stage for the beginning of the European cherry fruit fly oviposition. Two to three insecticide applications were usually applied against $R$. cerasi, yearly. Insecticides used were mainly neonicotinoids and pyrethroids in a spray volume of $1.6-1.7 \mathrm{~T} /$ ha and $0.8-1 \mathrm{~L} /$ tree. Tractor-sprayings 
were conducted early in the morning (sunrise) or late in the afternoon (after sunset). The main problem related to the insecticide applications is the fact that sprayings of late ripening cultivars during color break may coincide with harvest time of the early ripening ones planted in the next row. The risk of insecticide drift to neighboring rows is intensified by the weather conditions in spring that, in the respective area, are usually rainy and windy.

\subsection{Development of a Spatial Decision Support Tool for the European Cherry Fruit Fly}

The Spatial Decision Support System (SDSS) that we developed is illustrated in Figures 2 and 3 and a list of the terminology accompanied with their definition is provided in Table 2. The SDSS is composed of two algorithms that lead to a) the timing for the deployment of the trapping network in the field and b) decision(s) regarding timing of insecticide application against the European cherry fruit fly and the spatial location of application ("precision targeting"). The foundation of the first algorithm is a day degree model based on the lower developmental thresholds estimated by Baker and Miller [26] and the biofix (diapause termination) estimated from data of Papanastasiou and co-workers [6]. The lower developmental threshold (LDT) for the cherry fruit fly has been estimated at $5{ }^{\circ} \mathrm{C}$. The Day Degree buffer $(\mathrm{DDb})$ has also been calculated at $32^{\circ} \mathrm{C}$. Based on the first algorithm (Figure 2), when $\mathrm{DD}$ on a given date reaches or exceeds the $\mathrm{DDb}$, the trap network should be installed in the field. Timing of traps installation is quite important for the European cherry fruit fly, since the sticky traps used may accumulate debris of petals and sepals at the end of blossoming that diminish efficacy and dramatically increase trapping cost (labor for service, and replacement and use of new stick boards). The second algorithm is based on inputs regarding sweet cherry trees phenological stage, harvest dates and pre-harvest intervals for each cherry cultivar (calculated based on values from the last 3 years), estimation of the percentage of traps with captures (at least one R. cerasi adult), the parameter "flies per trap per day" and assessment of the spatial distribution of the fly's captures within the experimental plot. The algorithm's output defines the exact areas within each orchard where insecticidal application against $R$. cerasi should be applied, also taking into account the ripening stage of each cultivar.

Table 2. Explanatory input list of terms and symbols used in the SDSS algorithm for the control of the European cherry fruit fly.

\begin{tabular}{cc}
\hline Term/Symbol/Abbreviation & Definition \\
\hline DD & Day Degrees $=\Sigma($ Tmax - Tmin $) / 2-5$ \\
LDT/Biofix & Lower developmental threshold $=5{ }^{\circ} \mathrm{C}$ \\
DDb & Day Degrees buffer $=32{ }^{\circ} \mathrm{C}$
\end{tabular}




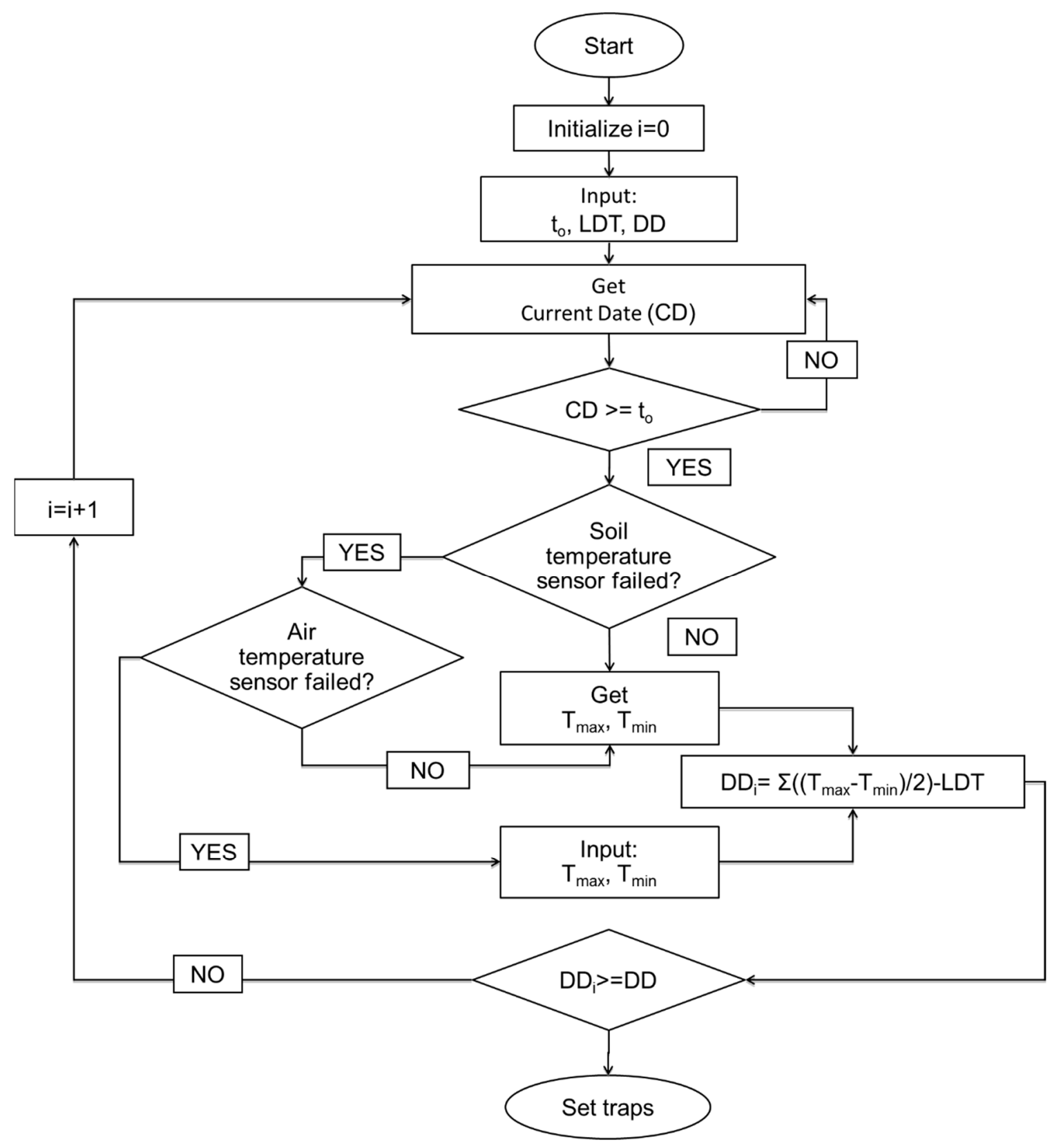

Figure 2. Algorithm of the DSS for trapping cherry fruit fly.

\subsection{Preparatory Monitoring in the Year 2014}

Since there was no available information regarding $R$. cerasi phenology in the area, the year before DSS field testing, we intensively monitored the fly populations in the area. To do so, we set a grid of 4 yellow Rebell amarillo ${ }^{\circledR}$ traps in each of the two experimental orchards (Orchards \#1 and \#2) on 19 April 2014. Orchard \# 3 was not available by that time (Figure 1). We also set 3 Rebell traps in individual, non-commercial (back-yard) cherry trees which did not receive any kind of control treatment. These trees served as reference (control) during the preparatory year. Rebell traps were monitored once a week until 4 July 2014, when two consecutive inspections with zero captures were observed (i.e., the end of the adult population). 


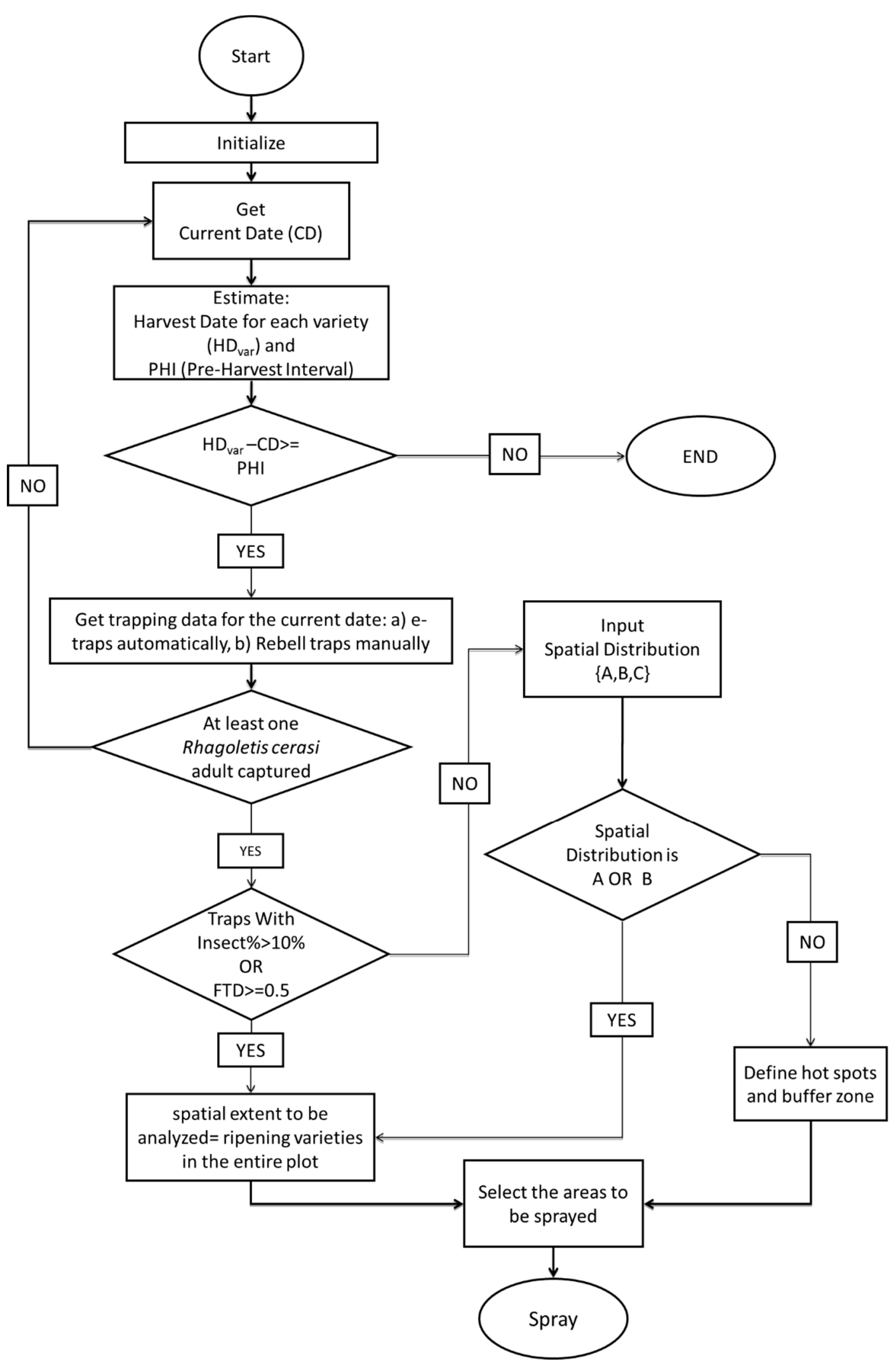

Figure 3. Algorithm of the DSS for spraying for the control of the cherry fruit fly. Data regarding adult captures were obtained manually. Potential future use of e-traps will allow automatic uploads of pictures to servers. 


\subsection{Implementation of the DSS in the Year 2015}

\subsubsection{Selection of Experimental Orchards}

The commercial cherry orchards selected in our study were divided in plots where spraying decisions regarding the control of $R$. cerasi were taken by the producer (IPM-adopted methodology) without any interference or advice by our group (Conventional plots- "Conv. Plots") and in plots where any decision regarding the control of R. cerasi was taken solely based on the developed DSS (DSS plots). In all experimental orchards, sweet cherry trees were mapped, and each cultivar was digitized including coordinates and ripening period (Figure 4). Orchard \#1 was considered as a block with 2 plots (0.7 ha each), one DSS and one conventional (Figure 5A). Orchard \#2 was divided in 2 blocks of 1 ha each. The two blocks included one DSS and one conventional plot ( 0.5 ha each) (Figure 5B). Orchard \#3 was also considered a block with 2 plots (DSS and conventional, 0.7 ha each) (Figure 5C). A buffer zone of two rows was considered between DSS and conventional plots. As a reference (control site), four additional intercropped, mixed, organic farms with cherry trees laying close to the experimental farms (situated $3.8 \mathrm{~km}$ east from Orchard \#3), were included in our study. The organic farms, apart from cherry trees, comprised of apples, pears, apricots, peaches and plums. Intercropping between tree rows included strawberries and vegetables (tomatoes, cucumbers, aubergines, etc.).

\subsubsection{Deployment of Trap Network, Population Monitoring and Spraying Decisions}

Based on the algorithm of the DSS for initiating trapping, a grid of 20 Rebell traps was deployed in each DSS and conventional plot. The accumulation of 400 Day Degrees, starting on 1 February 2015, was achieved on 26 April 2015, and this was considered the time point for traps establishment. A total of 160 Rebell traps were deployed in the 8 experimental plots ( 20 per plot). An additional grid of 22 Rebell traps was placed in the organic farms. Their density in each farm was adjusted according to the number of existing cherry trees. Despite the fact that $R$. cerasi management in conventional plots was performed according to IPM-adopted practices by the farmers with no additional consultation by our team, we decided to set up traps in order to draw a better picture regarding the pest's activity inside the experimental orchards. The geographic coordinates of each trap were collected upon installation, allowing precise mapping of the spatial distribution of cherry fruit fly trapping in each plot. The traps were inspected every 2-3 days until 19 June 2015, when the latest ripening cultivar was harvested. Capture data were uploaded on the same date of collection to the DSS platform.

Cherry fly population management in the conventional plots was performed according to the IPM-adopted practices with no intervention or provision of any kind of information by the field scientists. DSS plots management was completely based on the indications provided by the tested algorithms (Figures 2 and 3). When there was a decision for spraying, growers were guided on when and where to spray. The concentration of active ingredient (deltamethrin) in spraying volume was according to the product's instructions, and the quantity of insecticide sprayed per hectare in DSS plots and conventional plots was the same (1.6-1.7 T/ha).

\subsubsection{Estimation of Fruit Infestation Rates}

During the harvest period, we performed three fruit samplings (20 and 27 May 2015 and 9 June 2015) to estimate $R$. cerasi infestation rates. Samplings comprised of 100 randomly collected cherries of ripening cultivars from all conventional and DSS plots. We also collected 100, 100 and 50 cherries on the same dates, respectively, from the reference organic farms. Cherries were transferred to the laboratory $\left(25^{\circ} \mathrm{C}, 50 \% \pm 10 \% \mathrm{RH}\right.$ and photoperiod $\left.14 \mathrm{~L}: 10 \mathrm{D}\right)$ and placed in plastic containers on a layer of sterilized sand to facilitate pupation. Pupae were daily collected using soft forceps. 

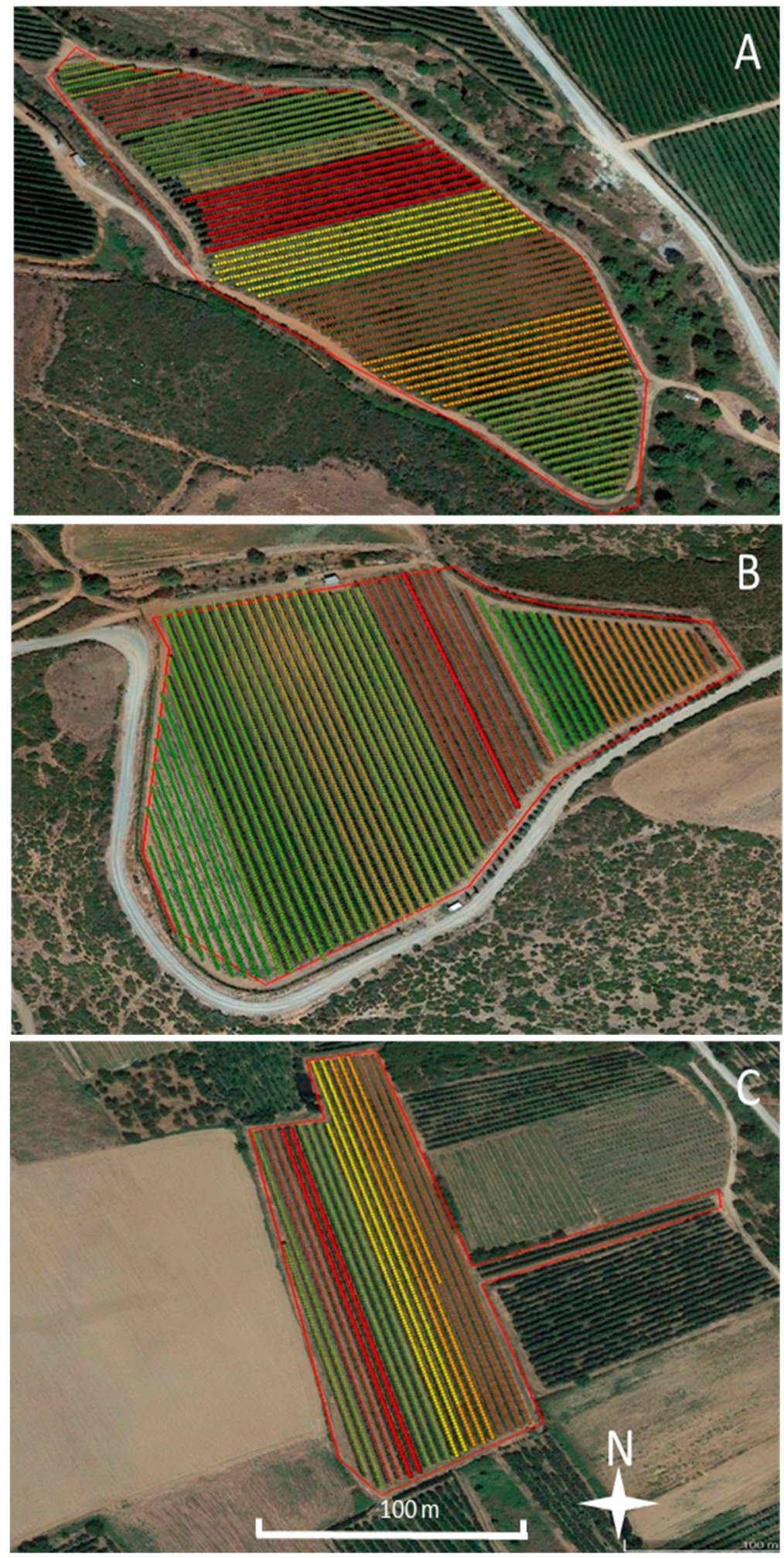

Figure 4. Digitization of different cherry cultivars (location and ripening period) in commercial cherry orchards used in our study. Different colors indicate different cherry cultivars. (A) Orchard \#1, (B) Orchard \#2, and (C) Orchard \#3. 

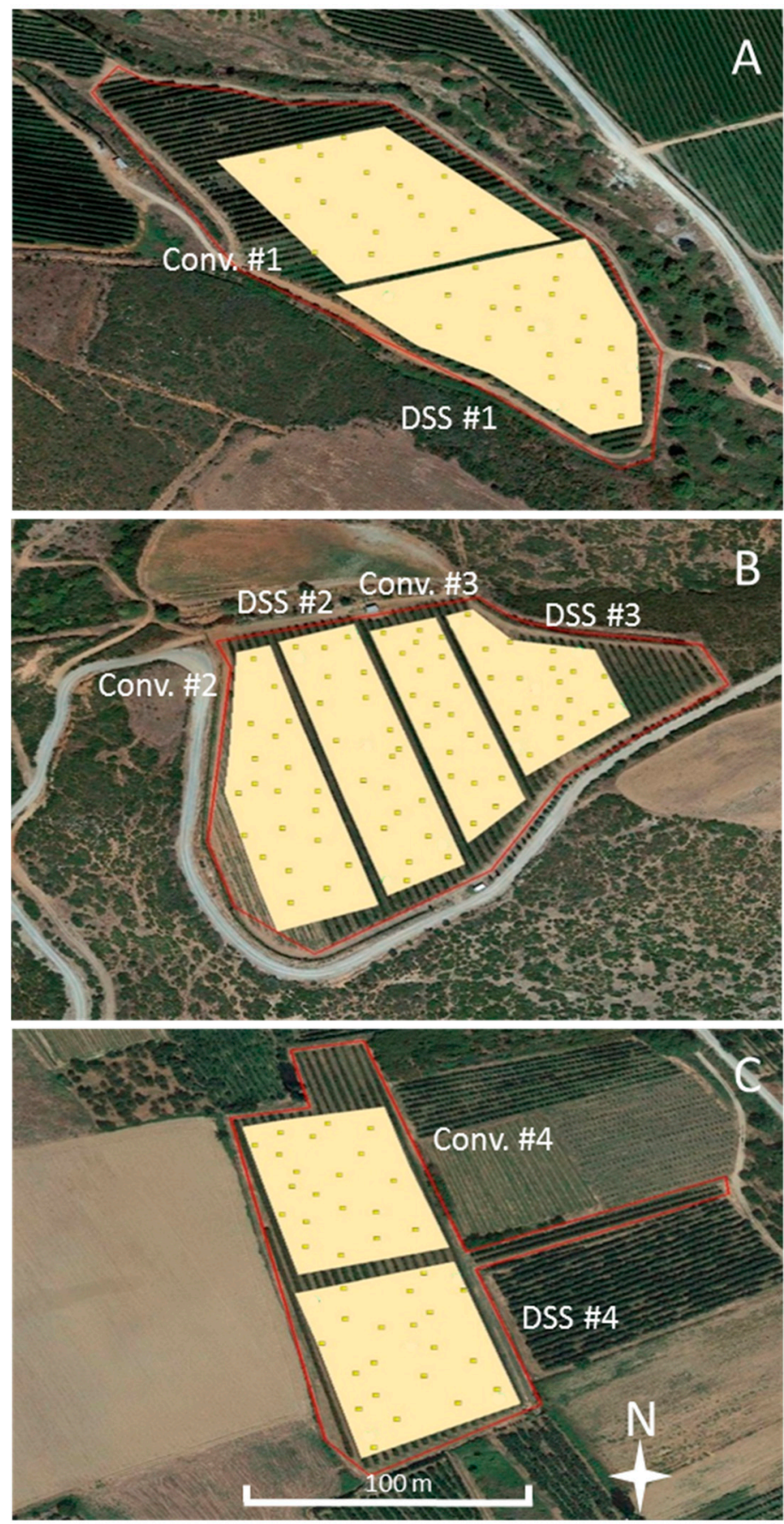

Figure 5. Digitization of coventionally treated (Conv.) and DSS treated plots in commercial cherry orchards. Yellow points indicate the position of Rebell traps. (A) Orchard \#1, (B) Orchard \#2, and (C) Orchard \#3.

\subsubsection{Cherry Fruit Insecticide Residual Levels}

Parallel to the fruit samplings for the estimation of infestation rates, a subset of 100 cherries was also collected on each sampling date for the evaluation of insecticide residues on the fruit. Assessment of residues was focused on the active compound deltamethrin, a pyrethroid which was used in all cases (either managed conventionally or with the DSS) to control cherry fruit fly adults. Although neonicotinoids are also certified for the control of R. cerasi, none of the growers applied another active ingredient than deltamethrin during the experimental period. Cherries from each sampling date and plot were pooled and homogenized to $300 \mathrm{~g}$ of pulp, using a blender. The pulp was then 
chemically solubilized and diluted in organic solvent, and chemical components separated via liquid chromatography [27]. The analysis was conducted in the laboratory of Analytical Chemistry of the Department of Agriculture, Crop Production and Rural Environment at the University of Thessaly.

\subsection{Data Analysis}

Data analyses were performed using the statistical software SPSS 24.0 (SPSS Inc., Chicago, IL, U.S.A.). Differences in adult $R$. cerasi captures among treatments in years 2014 and 2015 were estimated with a non-parametric independent samples Kruskal-Wallis H-test followed by the Mann-Whitney $\mathrm{U}$ criterion for pairwise comparisons. The same procedure was followed for the analysis of data concerning adult flight duration defined by first and last capture for the two consecutive years. In all cases, Bonferroni corrections were applied to adjust $p$-values for pairwise comparisons. The effect of the time period that traps remained in the orchards on the number of adult captures among treatments was assessed through binary logistic regression. Spatial analyses of the cherry fly adult populations were performed using Surfer ${ }^{\circledR}$ (Golden Software, Inc., Colorado, USA) following kriging interpolation. This procedure provides distributional estimates based on capture data and spatial continuity. Semiovariography was employed to infer on spatial patterns. Thematic spatial maps were considered for the application of spraying, depicting cherry fruit fly population densities within each experimental plot.

\section{Results}

\subsection{Population Trends During the Year 2014}

Mean adult cherry fruit fly captures and mean period with trap captures in the commercial farms and the control cherry trees during the preliminary monitoring in 2014 is shown in Table 3. Adult captures were significantly lower in commercial orchards than in traps placed in control unsprayed trees (Mann-Whitney $\mathrm{U}=0.5, p<0.05$ ). The adult flight period did not differ between commercial orchards and reference trees (Mann-Whitney $\mathrm{U}=3, p>0.05$ ); however, adult captures in the control site may have started approximately one or two weeks earlier than trap deployment. This was evident by the direct start of adult captures, right after trap placement on the reference cherry trees, with no previous registers of zero captures. Apparently, the deployment of a trap network on reference cherry trees was delayed and the onset of adult flight was not precisely determined in 2014.

Table 3. Mean number of adult Rhagoletis cerasi captures and mean period with captures, per trap, in commercial orchards and reference unsprayed cherry trees, during 2014.

\begin{tabular}{ccccc}
\hline Treatments & No of Traps & $\begin{array}{c}\text { Mean Captures } \\
\text { (Adults } \pm \text { SE) }\end{array}$ & $\begin{array}{c}\text { Mean Period with } \\
\text { Captures (Days } \pm \text { SE) }\end{array}$ & $\begin{array}{c}\text { \% of Traps } \\
\text { with Captures }\end{array}$ \\
\hline $\begin{array}{c}\text { Commercial orchards } \\
\text { Reference trees }\end{array}$ & 8 & $2.25 \pm 0.88 \mathrm{a}$ & $33.5 \pm 8.97 \mathrm{a}$ & 50 \\
\end{tabular}

\subsection{Implementation of the DSS During the Year 2015}

The Rebell trap network was set according to the Decision Support System (DSS) (Figure 2). The accumulation of Degree Days (DD) started on 1 February 2015. The accumulation of 400 DD was achieved on 26 April 2015, and this is when the establishment of our trap network (160 Rebell traps) was completed. The successful implementation of the DSS for setting the trap network is supported by the first R. cerasi adult capture recorded on 30 April 2015.

The population trends of $R$. cerasi in both the DSS and conventionally treated commercial orchards, and in the reference organic mixed farms during 2015 are shown in Table 4 and Figures 6 and 7. Adult captures during 2015 were extremely low in all plots of the commercial orchards, not exceeding 0.4 flies/trap during the whole experimental period (Figure 6). Average adult captures were significantly higher in the reference organic orchards than the commercial orchards (Reference vs. DSS: 
Mann-Whitney $U=16.5, p<0.001$; Reference vs. Conventional: Mann-Whitney $U=14.5, p<0.001)$. Rhagoletis cerasi captures were similar between Conventional and DSS treated plots (Mann-Whitney $\mathrm{U}=151, p>0.05$ ). Adult flight period (evidenced by trap captures) was significantly longer in the reference orchards compared to commercial orchards (Reference vs. DSS: Mann-Whitney U $=54, p<$ 0.001; Reference vs. Conventional: Mann-Whitney $\mathrm{U}=24, p<0.001)$. The flight period was similar between DSS and Conventional plots (Mann-Whitney $\mathrm{U}=2749.5, p=0.044$ ). Additional insecticide applications in the Conventional plots neighboring DSS plots, different pruning systems between commercial orchards and reference organic farms, as well as the inclusion of other fruit trees and intercropping methods in the latter may account for the longer flight period of $R$. cerasi and the higher percentage of traps with captures in the organic farms.

Table 4. Mean number of adult Rhagoletis cerasi captures and mean period with captures, per trap, in conventional and DSS plots of the commercial cherry orchards and in the reference organic farms during 2015.

\begin{tabular}{|c|c|c|c|c|}
\hline Experimental Plots & No of Traps & $\begin{array}{l}\text { Mean }{ }^{1} \text { Captures } \\
\text { (Adults } \pm \text { SE) }\end{array}$ & $\begin{array}{l}\text { Mean }{ }^{1} \text { Period with } \\
\text { Captures (Days } \pm \text { SE) }\end{array}$ & $\begin{array}{c}\% \text { of Traps } \\
\text { with Captures }\end{array}$ \\
\hline DSS 1 & 20 & $0.00 \pm 0.00$ & - & 0 \\
\hline DSS 2 & 20 & $1.10 \pm 0.22$ & $6.67 \pm 1.92$ & 75 \\
\hline DSS 3 & 20 & $1.05 \pm 0.29$ & $5.00 \pm 0.91$ & 50 \\
\hline DSS 4 & 20 & $0.05 \pm 0.05$ & 2.00 & 5 \\
\hline DSS total & 80 & $0.55 \pm 0.11 \mathrm{a}$ & $5.85 \pm 0.17 a$ & 32.5 \\
\hline Conventional 1 & 20 & $0.10 \pm 0.07$ & $3.00 \pm 1.00$ & 10 \\
\hline Conventional 2 & 20 & $0.15 \pm 0.08$ & $4.00 \pm 1.53$ & 15 \\
\hline Conventional 3 & 20 & $1.00 \pm 0.33$ & $6.33 \pm 2.74$ & 45 \\
\hline Conventional 4 & 20 & $0.05 \pm 0.05$ & 2.00 & 5 \\
\hline Conventional total & 80 & $0.325 \pm 0.10 \mathrm{a}$ & $5.13 \pm 1.68 \mathrm{a}$ & 18.75 \\
\hline Organic farms & 22 & $73.73 \pm 23.91 b$ & $26.55 \pm 2.74 b$ & 100 \\
\hline
\end{tabular}

${ }^{1}$ Means within a column followed by the same letter do not differ significantly (Mann-Whitney U-test with Bonferroni correction, adjusted $p=0.017$ ).

\subsection{Decisions on When and Where to Spray}

The "when and where" to spray algorithm is presented in Figure 3. The harvest date for each cultivar was set according to the harvest dates of the last three years (data given by the producer). In order to avoid pesticide residues on the fruit, it was pre-decided that cultivars with a shorter pre-harvest period to the one recommended by the pesticide manufacturer would not be sprayed in spite of R. cerasi being caught in the orchard. This scenario was not met in any of the four DSS plots tested.

From the onset of adult $R$. cerasi captures in the traps, the percentage of traps with adults and the FTD was daily calculated. In the commercial Orchard \#2 (DSS 2 and 3) the percentage of traps capturing adults exceeded $10 \%$ on 6 May 2015 and, thus, according to the DSS, the grower sprayed the entire plot. The decision was also supported by the observed spatial patterns (Figure 8). Although, the spatial distribution of captures was in fact clumped on 6 May 2015, it switched to random on May 8 and 11. Based on this situation, the farmer sprayed the entire orchard on 11 May 2015, instead of spraying hot spots. 

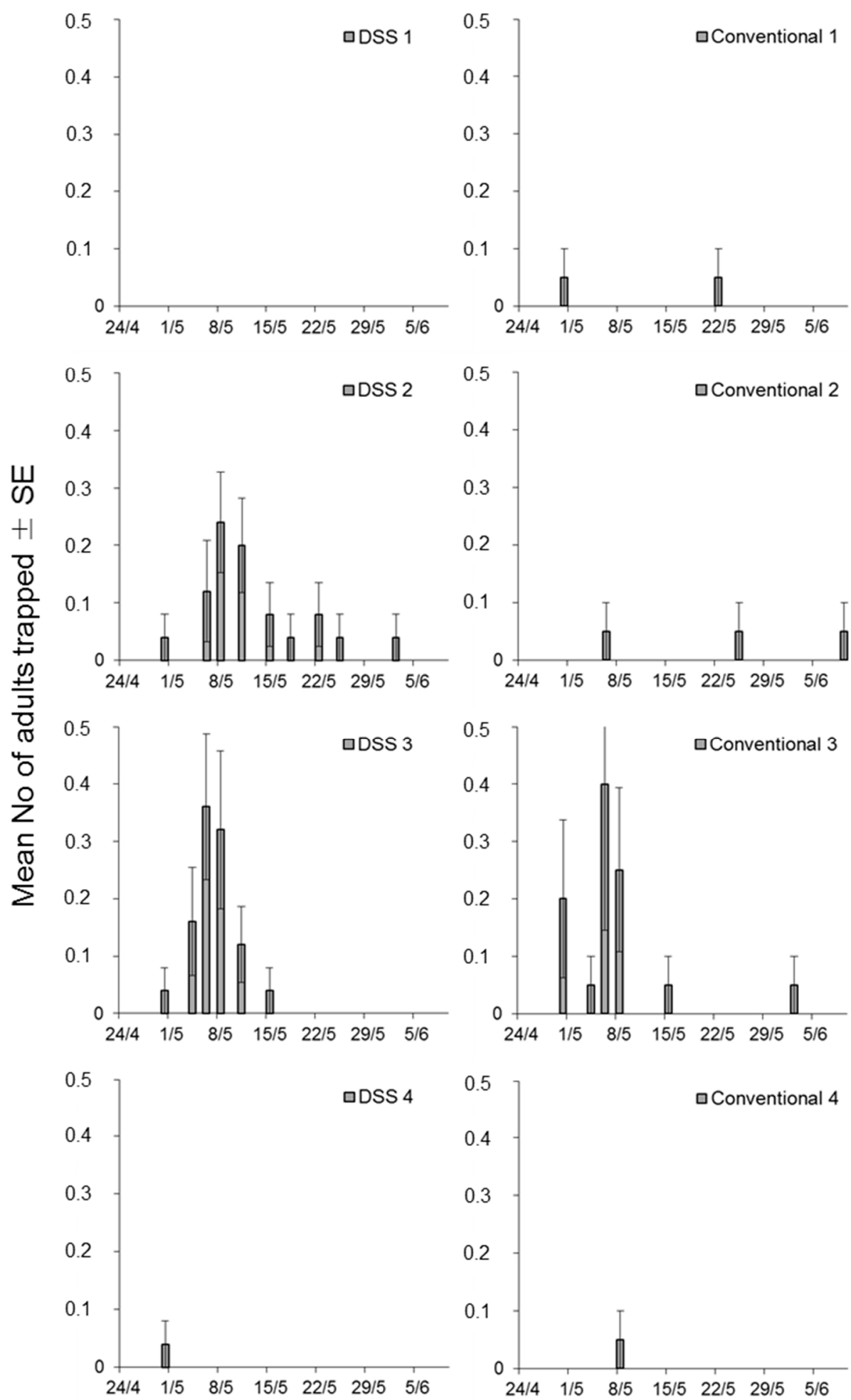

Dates 2015

Figure 6. Trapping trends of Rhagoletis cerasi during the cherry maturing period of 2015 in the DSS-supported and Conventional experimental plots.

In the four conventional plots, sprayings were performed according to farmers' IPM-based schedule. Conventional plots 1, 2 and 3 received two cover sprays on 30 April 2015 and 11 May 2015, respectively. Conventional plot 4 received two cover sprayings on 28 April and 8 May 2015. 
Farmers made spraying decisions for each conventional plot without the involvement of any DSS and considering the IPM-based management protocol applied by almost all farmers in the area.

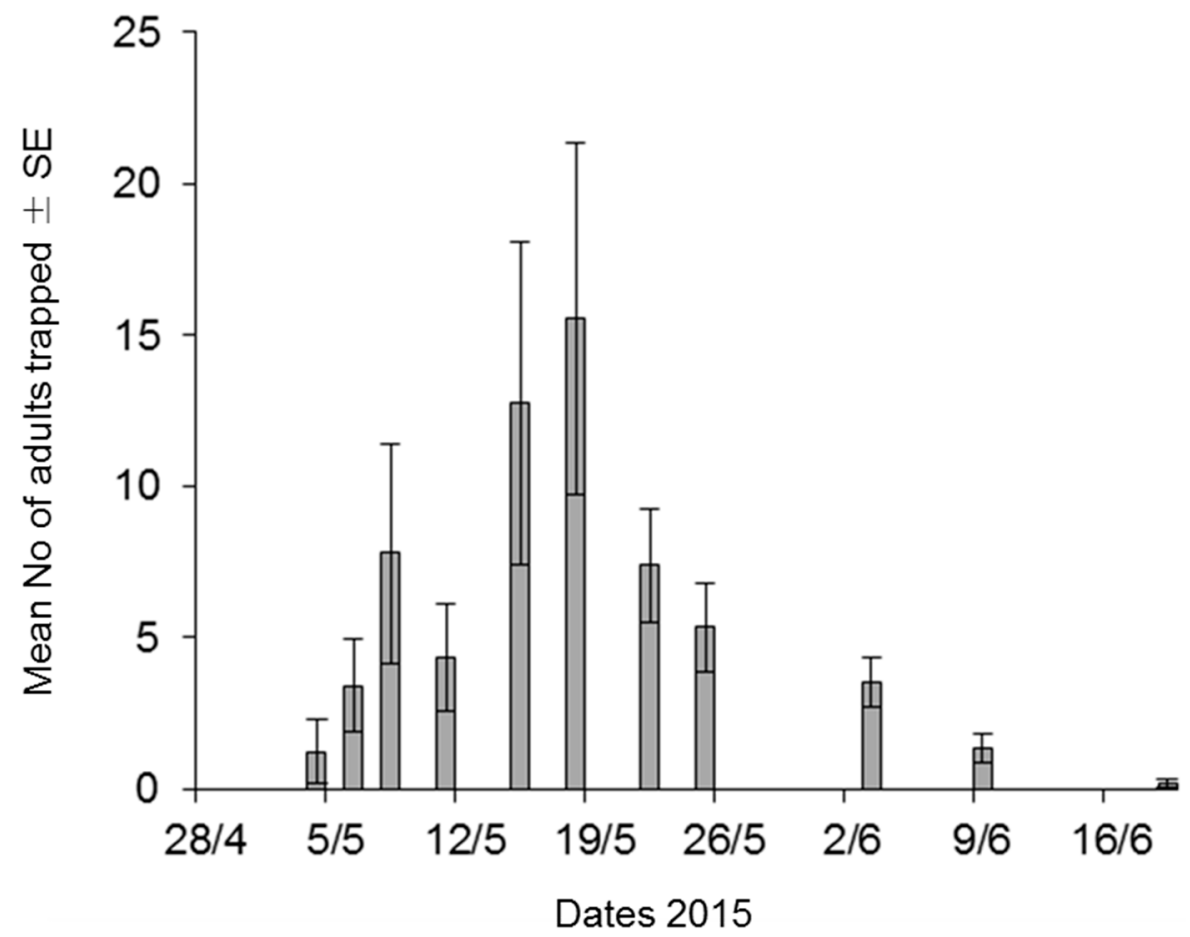

Figure 7. Trapping trends of Rhagoletis cerasi during the cherry maturing period of 2015 in the reference organic cherry farms.

\subsection{Fruit Infestation Rates}

Sampling of cherries from the DSS-managed and conventional managed plots on 20 and 27 May and 9 June revealed zero infestation. However, fruit sampling during the same dates from the reference organic cherry farms showed $21.5 \%, 6.4 \%$ and $8.8 \%$ infestation levels, respectively. Infestation rates in the organic farms were well above the acceptable fruit damage level $(2 \%)$.

\subsection{Insecticide Residues}

Liquid chromatographic analysis revealed that residues of deltamethrin were below the Maximum Residue Limits (MRLs $=0.2 \mu \mathrm{g} / \mathrm{gr}$ ) in all DSS and conventional plots tested (Table 5).

Table 5. Insecticide deltamethrin residue levels of cherry cultivars collected in different plots during cherry harvesting.

\begin{tabular}{|c|c|c|c|c|}
\hline Plot & Cherry Cultivar & Collection Date & Insecticide Residue Level & $\begin{array}{c}\text { Deltamethrin } \\
\text { Concentration }(\mu \mathrm{g} / \mathrm{gr})\end{array}$ \\
\hline DSS 1 & Ferrovia & $27 / 5 / 2015$ & Below detection level & - \\
\hline Conv. 1 & Samba & $27 / 5 / 2015$ & Below detection level & - \\
\hline DSS 2 & Kordia & 9/6/2015 & Below MRL ${ }^{1}$ & 0.069 \\
\hline DSS 3 & Giant red & $27 / 5 / 2015$ & Below detection level & - \\
\hline Conv. 3 & Vasiliadi & 9/6/2015 & Limits of detection & 0.016 \\
\hline DSS 4 & Larian & $9 / 6 / 2015$ & Below detection level & - \\
\hline Conv. 4 & Larian & $9 / 6 / 2015$ & Below detection level & - \\
\hline
\end{tabular}



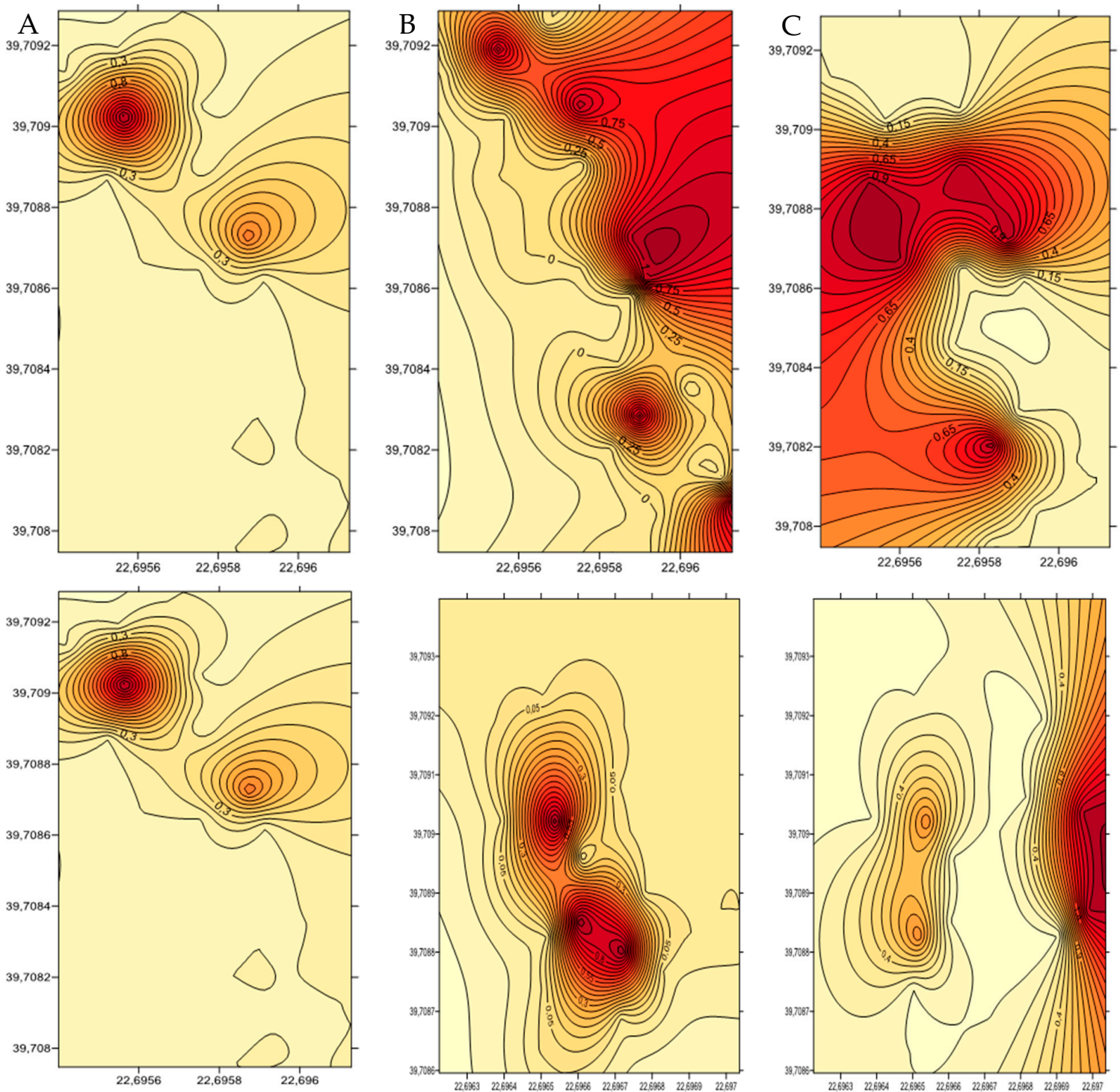

Figure 8. Spatial patterns of adult captures in DSS 2 (first row) and DSS 3 (second row) on 6 May 2015 (A), 8 May 2015 (B) and 11 May 2015 (C). The clumped distribution of captures at the beginning of the flight period was lost during the subsequent sampling dates in DSS 2. However, the number of captures was very low in DSS 3 and no solid conclusion could be extracted regarding the spatial distribution of captures. Intense red color indicates higher capture densities, while lines refer to the estimated density of adults. Captures in following days were very low.

\section{Discussion}

Overall, the results of this first implementation of a decision support system in cherry orchards to control a key pest, was highly successful. The implementation of our developed DSS for the management of cherry fruit fly in Greece resulted in a dramatic reduction of insecticide applications, when contrasted conventional management applied in the area. On average, the reduction of pesticide applications to control the cherry fruit fly in DSS-managed plots is estimated to be of $75 \%$ (one-half per plot in DSS treated compared to two applications per plot in conventionally treated plots). Applying the developed DSS kept $R$. cerasi populations at minimal levels and assured zero infestation on fruits. These values were similar to those of the conventionally treated plots and much lower compared to non-treated reference organic farms in the same area. Although $R$. cerasi is widespread and abundant in Agia, as evidenced during 2014 and by captures and damage in reference orchards during 2015, the 
density of the fly's population in the commercial orchards used in this study was low throughout 2015. The frequent application of pesticides and the thorough harvesting (because of the high prices of sweet cherries in the market) exercised in commercial orchards may explain these population trends. On the other hand, the slow mobility of adult cherry flies cannot bring feral flies from long distances to commercial orchards. Regardless of the population patterns in commercial orchards, a well-designed and structured population monitoring, as well as a strategic pest management approach is required to assure low $R$. cerasi population densities and to reduce fruit infestation levels to zero.

Both parts of the DSS algorithm performed well in field conditions leading to sound decisions. By adopting a simple degree day model, we managed to bring installation of the trapping network quite close to the beginning of adult $R$. cerasi flight in the wild, reducing the cost of population monitoring (e.g., labour for additional inspections, more traps used because of debris accumulations, etc.).

Phenological patterns of the European cherry fruit fly may be highly variable in different climatic conditions and, therefore, the developed algorithm should be locally adapted [6]. It is well documented that $R$. cerasi has developed a remarkable ability to adjust adult emergence in the wild in order to coincide with ripening periods of the host fruit [28]. Local adaptation and plastic responses of dormant pupae are key components to regulate adult emergence in the wild [29]. The short list of hosts, univoltinism and the relatively narrow periods of fruit ripening makes $R$. cerasi vulnerable to local extinction if timing of adult occurrence does not follow fruit ripening. Flight activity onset/end, duration and peak in the experimental area is typical for a lowland Greek $R$. cerasi population [6].

Working on commercial orchards we had to assure zero infestation and, hence, several inputs in the algorithm were set at very low levels. Nonetheless, executing the DSS led to an impressive reduction of insecticide applications. Systematic and strategically implemented IPM activities based on biotic, abiotic and other inputs (e.g., properties of the pesticides selected) may have major impact on the efficacy of pest management, assuring minimal chemical footprints on both the environment and the harvested product. Besides determining frequency of interventions, a thorough spatial analysis of adult captures is an important element of the developed DSS since it defines the extent of the area within the orchard to be treated. Rhagoletis cerasi populations tend to form clusters in the wild, closely following the spatial patterns of fruit availability and ripening $[15,19,20]$. Understanding this spatial behavior and utilizing the on-farm behavior and dispersion patterns of $R$. cerasi will undoubtedly optimize the site-specific level of IPM systems [1,30]. Spatial analysis to drive pest management decisions is more important under low pest population densities since fruit flies tend to follow a clustered distribution under these densities [25,31]. Thus, more sophisticated systems that analyze the on-farm behavior of fruit flies considering additional factors (e.g., age and physiological status of females), such as the PESTonFARM model, can greatly enhance the management ability of our simple DSS, leading to sounder IPM decisions.

Reducing pesticide use against $R$. cerasi has not only long-term environmental and human health benefits, but economic as well. Considering labor cost, equipment utilization and consumables (e.g., pesticides) we estimated that the average cost of R. cerasi management in the DSS-treated plots was 3.25 times lower than that in the conventionally treated ones ( $€ 29.5$ and $€ 96.1$ per hectare, respectively). Such a reduction in costs is essential for medium, small size farmers who are the majority in sweet cherry production systems in Europe. Adopting and implementing a DSS, such as the one used in the current study, in broader area by growers' cooperatives may further increase the economic benefits by the farmers. On the other hand, archiving both inputs and outputs of the DSS may facilitate product certification, simplifying national and international fruit trading.

Most known DSSs (e.g., CottonLOGIC, SIRATC; reviewed in [32]), including the pioneering MedCila, operate at regional levels. Application of MedCila resulted in pesticide use reduction (including fewer applications and less plots treated) against the Mediterranean fruit fly, Ceratitis capitata, in citrus orchards in Israel [22]. The above system is operating at a regional level and provides recommendations that are generally accepted by the pest management decision makers. The implementation of the present DSS for the control of $R$. cerasi could be a key component in both 
regional and area-wide IPM programs. However, untreated scattered individual cherry trees and organic orchards may, in several cases, severely hamper the positive outcome of such an approach. These factors should be taken into account when designing and applying large scale programs.

One of the major issues in DSS used in modern pest management is the low acceptance level [24]. Particularly, farmers feel quite uncomfortable when recommended to abstain from spraying in fear of yield losses and economic damage. Moreover, in many cases they lack the scientific background to easily adopt novel practices. Working on commercial farms and involving farmers and managers in execution of the DSS outputs, as we have done in the current study, increased the levels of acceptance and directly confirmed the importance of these systems to provide a wealth of information that assist sound pest management decisions. Demonstrating and proving that the implementation of the DSS results in zero infestation levels is a cornerstone for cherry producers because of the value of their product and international trading opportunities.

Although the implementation of the DSS that we developed for this study was performed manually, using simple Excel sheets and the software Surfer ${ }^{\circledR}$, the results are impressive and robust. A fully computerized system that uses real time collection of field data to run the algorithms of the DSS will increase its acceptance by farmers and pest managers. An electronic trap to monitor adult $R$. cerasi populations has already been developed [33], as well as a network of sensors to acquire satisfactory meteorological data. Improvement, refinement and full adoption of user-friendly systems that automatically and in real time acquire, process and analyze field and other data, assisting pest management decisions, will further enhance the utility of the DSS we have developed. Along the above lines, efforts have been made to develop a software platform for the facilitation and effectiveness of insecticide applications against several fruit flies, including the European cherry fruit fly (http://fruitflynet.aua.gr). Such systems have been tested against other pests over the last decade [34,35], and may serve to further disseminate the advantages of DSS in fruit fly pest management.

Author Contributions: Conceptualization and methodology, N.T.P., D.N., M.A.M. and A.S.; investigation and formal analysis C.S.I., S.A.P, N.T.P. and K.D.Z.; writing-original draft preparation, N.T.P., S.A.P. and C.S.I.; writing—review and editing, N.T.P., D.N., M.A.M., A.S., C.S.I., S.A.P., and K.D.Z.; supervision, N.T.P.

Funding: The EU through the two-year Project FruitFlyNet/II-B/2.1/0865/ENPI CBC MED/EU/GRAND No 2438/49/30.12.2013 financed this study. The project "A Location-aware System for Fruit Fly Monitoring and Pest Management Control, FruitFlyNet" is part of the ENPI CBC Mediterranean Sea Basin Programme. This cross-border cooperation (CBC) multilateral initiative, funded by the EU under the European Neighborhood Partnership Instrument (ENPI), gathers 14 countries from both shores of the Mediterranean with a view to address common challenges in fields such as support for economic clusters and SMEs, environmental sustainability, enhancement of cultural heritage, people-to-people cooperation, and local governance. More information on this program is available on its website: www.enpicbcmed.eu.

Acknowledgments: To the Tsaknakis, Apostolou and Tsiagalis families, for allowing to perform the study in their cherry growing commercial orchards.

Conflicts of Interest: The authors declare no conflict of interest.

\section{References}

1. Papadopoulos, N.T.; Lux, S.A.; Köppler, K.; Beliën, T. Invertebrate and Vertebrate Pests: Biology and Management. In Cherries: Botany, Production and Uses; CABI: Wallingford, UK, 2017; pp. 305-337.

2. Augustinos, A.; Moraiti, C.; Drosopoulou, E.; Kounatidis, I.; Mavragani-Tsipidou, P.; Bourtzis, K.; Papadopoulos, N. Old residents and new arrivals of Rhagoletis species in Europe. Bull. Entomol. Res. 2019, 1-12. [CrossRef] [PubMed]

3. Cini, A.; Anfora, G.; Escudero-Colomar, L.A.; Grassi, A.; Santosuosso, U.; Seljak, G.; Papini, A. Tracking the invasion of the alien fruit pest Drosophila suzukii in Europe. J. Pest Sci. 2014, 87, 559-566. [CrossRef]

4. Barringer, L. First record of the European cherry fruit fly, Rhagoletis cerasi (Linnaeus)(Diptera: Tephritidae), in North America. Insecta Mundi 2018, 0622, 1-4.

5. Wakie, T.T.; Yee, W.L.; Neven, L.G. Assessing the Risk of Establishment of Rhagoletis cerasi (Diptera: Tephritidae) in the United States and Globally. J. Econ. Entomol. 2018, 111, 1275-1284. [CrossRef] [PubMed] 
6. Papanastasiou, S.A.; Nestel, D.; Diamantidis, A.D.; Nakas, C.T.; Papadopoulos, N.T. Physiological and biological patterns of a highland and a coastal population of the European cherry fruit fly during diapause. J. Insect Physiol. 2011, 57, 83-93. [CrossRef] [PubMed]

7. Daniel, C.; Grunder, J. Integrated management of European cherry fruit fly Rhagoletis cerasi (L.): Situation in Switzerland and Europe. Insects 2012, 3, 956-988. [CrossRef]

8. Köppler, K.; Peters, A.; Vogt, H. Initial results in the application of entomopathogenic nematodes against the European cherry fruit fly Rhagoletis cerasi L. (Diptera, Tephritidae). Bull. OILB SROP 2005, 28, 13-18.

9. Köppler, K.; Kaffer, T.; Vogt, H. Bait sprays against the European cherry fruit fly Rhagoletis cerasi: Status Quo \& Perspectives. In Ecofruit-13th International Conference on Cultivation Technique and Phytopathological Problems in Organic Fruit-Growing: Proceedings to the Conference from 18th February to 20th February 2008 at Weinsberg/Germany; Boos, M., Ed.; Fördergemeinschaft Ökologischer Obstbau eV (FÖKO): Weinsberg, Germany, 2008; pp. 102-108.

10. Herz, A.; Köppler, K.; Vogt, H.; Elias, E.; Peters, A.; Katz, P. Biological control of the cherry fruit fly, Rhagoletis cerasi L.(Diptera, Tephritidae) by use of entomopathogenic nematodes: First experiences towards practical implementation. In Ecofruit-12th International Conference on Cultivation Technique and Phytopathological Problems in Organic Fruit-Growing: Proceedings to the Conference from 31st January to 2nd February 2006 at Weinsberg/Germany; Boos, M., Ed.; Fördergemeinschaft Ökologischer Obstbau eV (FÖKO): Weinsberg, Germany, 2006; pp. 67-72.

11. Daniel, C.; Mathis, S.; Feichtinger, G. A new visual trap for Rhagoletis cerasi (L.)(Diptera: Tephritidae). Insects 2014, 5, 564-576. [CrossRef]

12. Katsoyannos, B.I.; Papadopoulos, N.T.; Stavridis, D. Evaluation of trap types and food attractants for Rhagoletis cerasi (Diptera: Tephritidae). J. Econ. Entomol. 2000, 93, 1005-1010. [CrossRef]

13. Boller, E.F.; Remund, U. Field feasibility study for the application of SIT in Rhagoletis cerasi L. in Northwest Switzerland (1976-79). In Fruit Flies of Economic Importance. Proceedings of the CEC/IOBC International Symposium Athens, November 1982; Calvalloro, R., Ed.; Balkema: Rotterdam, The Netherlands, 1983; pp. 366-370.

14. Brand, G.; Höhn, H.; Kuske, S.; Samietz, J. Management of European cherry fruit fly (Rhagoletis cerasi) with exclusion netting: First results. IOBC WPRS Bull. 2013, 91, 401-404.

15. Daniel, C.; Baker, B. Dispersal of Rhagoletis cerasi in commercial cherry orchards: Efficacy of soil covering nets for Cherry Fruit Fly control. Insects 2013, 4, 168-176. [CrossRef]

16. Leski, R. Studia nad biologia i ecologia nasionnicy tzresniowki Rhagoletis cerasi L. (Diptera: Trypetidae). Pol. Pismo Entomol. Ser. B 1963, 3, 153-240.

17. Kovanci, O.; Kovanci, B. Reduced-risk management of Rhagoletis cerasi flies (host race Prunus) in combination with a preliminary phenological model. J. Insect Sci. 2006, 6, 34. [CrossRef] [PubMed]

18. Samietz, J.; Graf, B.; Höhn, H.; Schaub, L.; Höpli, H. Phenology modelling of major insect pests in fruit orchards from biological basics to decision support: The forecasting tool SOPRA. EPPO Bull. 2007, 37, 255-260. [CrossRef]

19. Lux, S.A.; Michalska, K.; Wnuk, A.; Paliczuk, D.; Vogt, H.; Hernik, T.; Studnicki, M. Female age as a factor determining the patterns of tree canopy utilisation by the European cherry fruit fly, Rhagoletis cerasi. Int. J. Pest Manag. 2017, 63, 371-381. [CrossRef]

20. Lux, S. PESTonFARM-stochastic model of on-farm insect behaviour and their response to IPM interventions. J. Appl. Entomol. 2014, 138, 458-467. [CrossRef]

21. Pedigo, L.P.; Rice, M.E. Entomology and Pest Management; Waveland Press: Long Grove, IL, USA, 2014.

22. Cohen, Y.; Cohen, A.; Hetzroni, A.; Alchanatis, V.; Broday, D.; Gazit, Y.; Timar, D. Spatial decision support system for Medfly control in citrus. Comput. Electron. Agric. 2008, 62, 107-117. [CrossRef]

23. Cohen, Y.; Cohen, A.; Broday, D. A knowledge-based SDSS for medfly Area-Wide Control: Development, validation and effectiveness evaluation. In Decision Support Systems in Agriculture, Food and the Environment: Trends, Applications and Advances; IGI Global: Hershey, PA, USA, 2010; pp. 21-46.

24. Nestel, D.; Cohen, Y.; Shaked, B.; Alchanatis, V.; Nemny-Lavy, E.; Miranda, M.A.; Sciarretta, A.; Papadopoulos, N.T. An Integrated Decision Support System for environmentally-friendly management of the Ethiopian fruit fly in greenhouse crops. Agronomy 2019, 9, 459. [CrossRef] 
25. Sciarretta, A.; Tabilio, M.R.; Lampazzi, E.; Ceccaroli, C.; Colacci, M.; Trematerra, P. Analysis of the Mediterranean fruit fly [Ceratitis capitata (Wiedemann)] spatio-temporal distribution in relation to sex and female mating status for precision IPM. PLoS ONE 2018, 13, e0195097. [CrossRef]

26. Baker, C.R.B.; Miller, G.W. Effect of temperature on postdiapause development of four geographical populations of european cherry fruit fly (Rhagoletis cerasi). Entomol. Exp. Appl. 1978, 23, 1-13. [CrossRef]

27. Anastassiades, M.; Lehotay, S.J.; Štajnbaher, D.; Schenck, F.J. Fast and easy multiresidue method employing acetonitrile extraction/partitioning and "dispersive solid-phase extraction" for the determination of pesticide residues in produce. J. AOAC Int. 2003, 86, 412-431. [PubMed]

28. Moraiti, C.A.; Papadopoulos, N.T. Obligate annual and successive facultative diapause establish a bet-hedging strategy of Rhagoletis cerasi (D iptera: T ephritidae) in seasonally unpredictable environments. Physiol. Entomol. 2017, 42, 225-231. [CrossRef]

29. Moraiti, C.A.; Nakas, C.T.; Papadopoulos, N.T. Diapause termination of Rhagoletis cerasi pupae is regulated by local adaptation and phenotypic plasticity: Escape in time through bet-hedging strategies. J. Evol. Biol. 2014, 27, 43-54. [CrossRef] [PubMed]

30. Lux, S.A.; Wnuk, A.; Vogt, H.; Belien, T.; Spornberger, A.; Studnicki, M. Validation of individual-based markov-like stochastic process model of insect behavior and a "Virtual Farm" concept for enhancement of site-specific IPM. Front. Physiol. 2016, 7, 363. [CrossRef] [PubMed]

31. Papadopoulos, N.T.; Plant, R.E.; Carey, J.R. From trickle to flood: The large-scale, cryptic invasion of California by tropical fruit flies. Proc. R. Soc. B Biol. Sci. 2013, 280, 20131466. [CrossRef] [PubMed]

32. Arora, R.; Singh, B.; Dhawan, A. Theory and Practice of Integrated Pest Management; Scientific Publishers: Jodhpur, India, 2017.

33. Shaked, B.; Amore, A.; Ioannou, C.; Valdés, F.; Alorda, B.; Papanastasiou, S.; Goldshtein, E.; Shenderey, C.; Leza, M.; Pontikakos, C.; et al. Electronic traps for detection and population monitoring of adult fruit flies (Diptera: Tephritidae). J. Appl. Entomol. 2017. [CrossRef]

34. Pontikakos, C.M.; Tsiligiridis, T.A.; Yialouris, C.P.; Kontodimas, D.C. Pest management control of olive fruit fly (Bactrocera oleae) based on a location-aware agro-environmental system. Comput. Electron. Agric. 2012, 87, 39-50. [CrossRef]

35. Tsiligiridis, T.; Pontikakos, C.; Perdikis, D. Architectural issues of a location-aware system applied in fruit fly e-monitoring and spraying control. AGRIS LINE Pap. Econ. Inform. 2014, 6, 195-207.

(C) 2019 by the authors. Licensee MDPI, Basel, Switzerland. This article is an open access article distributed under the terms and conditions of the Creative Commons Attribution (CC BY) license (http://creativecommons.org/licenses/by/4.0/). 